\title{
The correlation between the Trendelenburg position and the stroke volume variation
}

\author{
Jin Hye Min, Sang Eun Lee, Hong Sik Lee, Young Keun Chae, Yong Kyung Lee, Yoo Kang, and Ui Jin Je
}

Department of Anesthesiology and Pain Medicine, Myongji Hospital, Goyang, Korea

Background: The stroke volume variation (SVV), based on lung-heart interaction during mechanical ventilation, is a useful dynamic parameter for fluid responsiveness. However, it is affected by many factors. The aim of this study was to evaluate the effects of SVV on Trendelenburg (T) and reverse Trendelenburg (RT) position and to further elaborate on the patterns of the SVV with position.

Methods: Forty-two patients undergoing elective surgery were enrolled in this study. Fifteen minutes after standardized induction of anesthesia with propofol, fentanyl, and rocuronium with volume controlled ventilation (tidal volume of $8 \mathrm{ml} / \mathrm{kg}$ of ideal body weight, inspiration : expiration ratio of $1: 2$, and respiratory rate of $10-13 \mathrm{breaths} / \mathrm{min}$ ), the patients underwent posture changes as follows: supine, T position at slopes of operating table of $-5^{\circ},-10^{\circ}$, and $-15^{\circ}$, and RT position at slopes of operating table of $5^{\circ}, 10^{\circ}$, and $15^{\circ}$. At each point, $S V V$, cardiac output (CO), peak airway pressure (PAP), mean blood pressure, and heart rate (HR) were recorded.

Results: The SVV was significant decreased with decreased slopes of operating table in $\mathrm{T}$ position, and increased with increased slopes of operating table in RT position $(\mathrm{P}=0.000)$. Schematically, it was increased by $1 \%$ when the slope of operating table was increased by $5^{\circ}$. But, the $\mathrm{CO}$ and PAP were significant increased with decreased slopes of operating table in $\mathrm{T}$ position, and decreased with increased slopes of operating table in $\mathrm{RT}$ position $(\mathrm{P}=0.045,0.027)$.

Conclusions: SVV is subjected to the posture, and we should take these findings into account on reading SVV for fluid therapy. (Korean J Anesthesiol 2014; 67: 378-383)

Key Words: Fluid therapy, Reverse Trendelenburg position, Stroke volume variation, Trendelenburg position.

Received: June 26, 2014. Revised: July 25, 2014. Accepted: August 11, 2014.

Corresponding author: Jin Hye Min, M.D., Ph.D., Department of Anesthesiology and Pain Medicine, Myongi Hospital, 55, Hwasu-ro 14beon-gil, Deogyang-gu, Goyang 415-270, Korea. Tel: 82-31-810-6200, Fax: 82-31-810-6209, E-mail: jhmin@kd.ac.kr

(c) This is an open-access article distributed under the terms of the Creative Commons Attribution Non-Commercial License (http:// creativecommons.org/licenses/by-nc/3.0/), which permits unrestricted non-commercial use, distribution, and reproduction in any medium, provided the original work is properly cited. 


\section{Introduction}

It is impossible to overestimate the importance of proper fluid management in anesthesiology, because it is helpful not only for lowering the postoperative morbidity but also for improving outcomes [1]. However, there are still no reliable methods for evaluating the volume status. The central venous pressure (CVP) and the pulmonary capillary wedge pressure (PCWP) reveal limitations in predicting the fluid responsiveness [2].

The recently, introduced methods for measuring the uncalibrated pulse contour-derived fluid responsiveness, provided the possibility of obtaining the dynamic data about fluid responsiveness. This is followed by many reports showing that its reliability is higher compared with other static parameters $[3,4]$. The stroke volume variation (SVV) is one of the dynamic parameters of preload. But, its applicability is somewhat limited. That is, it may be solely used for patients with mechanical ventilation, because it is based on regular changes in the intra-thoracic pressure (ITP) through mechanical ventilation. Therefore, such regular changes in the ITP depending on the arterial waveform deserve special attention. This leads to the speculation that the SVV can also be subjected to factors that may affect the ITP [5].

In clinical settings, the patients' postures may commonly be altered to optimize the surgical conditions. It may affect the ITP by shifting abdominal contents; this is notably seen in the Trendelenburg (T) or reverse Trendelenburg (RT) position [6-8]. Bu,t there are no established reports about the effects of these positions on the SVV.

Given the above background, we conducted this study to examine the effects of SVV depending on the posture, thus further elaborating on whether the patterns of changes in the SVV are associated with the slope of operating table.

\section{Materials and Methods}

The study protocol was approved by the Institutional Review Board of our medical institution. All of the patients submitted written informed consent. In the current study, we enrolled 50 adult patients with the American Society of Anesthesiologists (ASA) physical status I or II, who were scheduled for elective surgery. We excluded the patients with arrhythmia, valvular heart disease, coronary artery disease, ejection fraction of $<50 \%$, pulmonary disease, or body mass index $<18.5 \mathrm{~kg} / \mathrm{m}^{2}$ or $>30 \mathrm{~kg} / \mathrm{m}^{2}$.

When the patient arrived at operating room, they underwent routine monitoring of electrocardiogram, non-invasive blood pressure measurement, pulse oximetry, and Bispectral Index sensor measurement (BIS ${ }^{\circledR}$, Covidien, Mansfield, MA, USA). The anesthesia performed according to the standardized protocol. The patient was induced with intravenous propofol $2 \mathrm{mg} / \mathrm{kg}$, fentanyl $1 \mathrm{ug} / \mathrm{kg}$, and rocuronium $0.6 \mathrm{mg} / \mathrm{kg}$. After tracheal intuba- tion, the patients underwent volume-controlled ventilation with Avance (Datex-Ohmeda, WI, USA) at a tidal volume of $8 \mathrm{ml} /$ $\mathrm{kg}$ of ideal body weight, an inspiration: expiration ratio of $1: 2$, a respiratory rate of $10-13$ breaths/min without positive endexpiratory pressure, and the end-tidal carbon dioxide tension $\left(\mathrm{ETCO}_{2}\right)$ maintained at $30-35 \mathrm{mmHg}$. The patient's ideal body weight was calculated using the following formula: $\mathrm{men}=50+$ 0.91 (height $[\mathrm{cm}]-152.4$ ), women $=45.5+0.91$ (height $[\mathrm{cm}]$ - 152.4). The anesthesia was maintained with $1.5-2$ vol $\%$ of sevoflurane with BIS scores of 40-60. This was followed by the insertion of a 20 gauge catheter in the radial artery and the connection to the Flotrac ${ }^{\mathrm{TM}} /$ Vigileo $^{\mathrm{TM}}$ system with software version 4.0 (Edwards Lifescience, Irvine, USA). The stopcock was attached to the patients' mid-axillary line in the left 5th intercostal space, for which zeroing was done. This system shows stroke volume (SV) and cardiac output (CO) continuously from the pulse contour analysis every 20 seconds. The SVV represents the change in beat-to beat $\mathrm{SV}$, and it is calculated according to the following formula: $(S V V=[S V m a x-S V m i n] /[(S V m a x+S V-$ min) / 2]), where SVmax and SVmin represent the maximal and minimal SV, respectively. When the patient was hemodynamically stable for at least 15 minutes after induction, we recorded patients' hemodynamic variables in supine position $\left(\mathrm{T}_{0}\right)$. Then, the patient's posture was changed to the $\mathrm{T}$ position at slopes of an operating table of $-5^{\circ}\left(\mathrm{T}_{-5}\right),-10^{\circ}\left(\mathrm{T}_{-10}\right)$, and $-15^{\circ}\left(\mathrm{T}_{-15}\right)$, we performed recording after 5 minutes of each posture. After the operating table was turned back to $0^{\circ}\left(\mathrm{T}_{0}{ }^{\prime}\right)$ and its slope was changed to the head-up tilt at $5^{\circ}\left(\mathrm{T}_{5}\right), 10^{\circ}\left(\mathrm{T}_{10}\right)$, and $15^{\circ}\left(\mathrm{T}_{15}\right)$, we recorded the variables in the same manner. Lastly, the patient was returned to the supine position $\left(\mathrm{T}_{0}{ }^{\prime}\right)$. At each point, we recorded SVV, cardiac output (CO) from Flotrac ${ }^{\mathrm{TM}} /$ Vigileo $^{\mathrm{TM}}$ system (Edwards Lifescience, Irvine, USA) and peak airway pressure (PAP) from spirometry of the Avance (Datex-Ohmeda, WI, USA). The mean blood pressure (MBP) and heart rate (HR) were also recorded.

During the recording, the patient's maintenance fluid was crystalloid by the $4: 2: 1$ rules, and the ventilator mode and anesthetic depth also remained constant without any stimulus. When the patient had an increase of PAP to $30 \mathrm{cmH}_{2} \mathrm{O}$, required vasopressors during the posture change, or presented with arrhythmia, we discontinued the recording and withdrew the corresponding patient from the current study.

The changes in SVV were fitted in a linear mixed model, for which the intraclass correlation coefficient was estimated. To do this, we used the $\mathrm{R}$ version 3.0.3 (R: A language and environment for statistical computing, R Foundation for Statistical Computing, Vienna, Austria) and nlme library (Jose Pinheiro, Douglas Bates, SaikatDebRoy, DeepayanSarkar and the R Development Core Team [2013], nlme: Linear and Nonlinear Mixed Effects Models R package version 3.1-113). All data were 
expressed as mean \pm SD or the number of patients. The $\mathrm{P}<0.05$ was considered as statistically significant.

\section{Results}

In the current study, we enrolled a total of 50 patients. Among these patients, 8 patients were excluded from the analysis ( 3 patients required vasopressors, 1 patient developed arrhythmia, and 4 patients had 20\% difference in measurements after being placed in supine position three times). Therefore, we performed statistical analysis with the remaining 42 patients.

Baseline and demographic characteristics of the patients are shown in Table 1 . There were 31 patients with the ASA class II, 22 patients with hypertension, 10 patients with diabetes mellitus, and 4 patients with stroke. For the types of surgery, there were $24,10,4,2$, and 2 patients who underwent abdominal surgery, neurosurgery, orthopedic surgery, urologic surgery, and otorhinolaryngologic surgery, respectively.

The hemodynamic data of the patients are shown in Table 2.

The SVV was significant decreased in proportion to the $\mathrm{T}$ positions at slopes of operating table of $-5^{\circ},-10^{\circ}$, and $-15^{\circ}$, in contrast, it was increased in the RT positions at slopes of operating table of $5^{\circ}, 10^{\circ}$, and $15^{\circ}(\mathrm{P}=0.000)$. The SVV increased by $0.2 \%$ with the increased slope of operating table, and intra-

Table 1. Demographic Characteristics

\begin{tabular}{lc}
\hline Number of patients & 42 \\
Age $(\mathrm{yr})$ & $60.9 \pm 13.1$ \\
Sex $(\mathrm{M} / \mathrm{F})$ & $28 / 14$ \\
ASA classification (I/II) & $11 / 31$ \\
Weight $(\mathrm{kg})$ & $65.6 \pm 13.3$ \\
Height $(\mathrm{cm})$ & $163.3 \pm 10.2$ \\
Tidal volume (ml) & $487.7 \pm 61.9$ \\
Respiration rate $(/ \mathrm{min})$ & $11.50 \pm 0.9$ \\
\hline
\end{tabular}

Data are presented as means \pm SD or the number. class correlation coefficient was 0.71 (Fig. 1).

The CO and PAP were significantly increased with the T position and decreased with the RT position ( $\mathrm{P}=0.045,0.027)$. But, changes of MBP and HR did not have statistical significance $(\mathrm{P}=0.175,0.790)$.

\section{Discussion}

Our results showed that the SVV was increased with increased slopes of operating table in the RT position. However, it was decreased with decreased slopes in the T position. The error in this model was evenly distributed around zero, and it corresponded to a linear mixed model. Schematically, the SVV was increased by $1 \%$ when there was an increase in the slope of operating table by $5^{\circ}$.

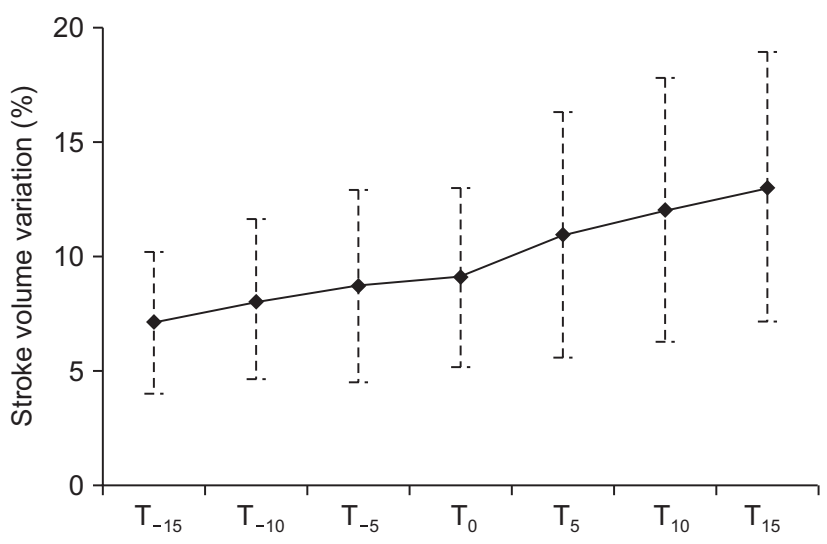

Fig. 1. Stroke Volume Variation according to Posture. Data are presented as means $\pm \mathrm{SD}$. SVV increased with increasing slope of operating table, and these were statistically significant $(P=0.000)$. SVV: stroke volume variation. $\mathrm{T}_{-15}$ : at $-15^{\circ}$ slope of an operating table in head-down tilt, $\mathrm{T}_{-10}$ : at $-10^{\circ}$ slope of an operating table in head-down tilt, $\mathrm{T}_{-5}$ : at $-5^{\circ}$ slope of an operating table in head-down tilt, $\mathrm{T}_{0}$ : at $0^{\circ}$ slope of an operating table in supine position, $\mathrm{T}_{5}$ : at $5^{\circ}$ slope of an operating table of $-5^{\circ}$ in head-up tilt, $\mathrm{T}_{10}$ : at $10^{\circ}$ slope of an operating table in head-up tilt, and $\mathrm{T}_{15}$ : at $15^{\circ}$ slope of an operating table in head-up tilt.

Table 2. Hemodynamic Data on Postural Changes in Trendelenburg Positions and Reverse Trendelenburg Positions

\begin{tabular}{|c|c|c|c|c|c|c|c|c|}
\hline & \multicolumn{4}{|c|}{ Trendelenburg positions } & \multicolumn{4}{|c|}{ Reverse Trendelenburg positions } \\
\hline & $\mathrm{T}_{0}$ & $\mathrm{~T}_{-5}$ & $\mathrm{~T}_{-10}$ & $\mathrm{~T}_{-15}$ & $\mathrm{~T}_{0}$ & $\mathrm{~T}_{5}$ & $\mathrm{~T}_{10}$ & $\mathrm{~T}_{15}$ \\
\hline SVV (\%) & $9.1 \pm 3.9$ & $8.7 \pm 4.2$ & $8.1 \pm 3.5$ & $7.1 \pm 3.1$ & $9.1 \pm 3.9$ & $10.9 \pm 5.4$ & $12.0 \pm 5.7$ & $13.0 \pm 5.9$ \\
\hline $\mathrm{CO}(\mathrm{L} / \mathrm{min})$ & $4.8 \pm 1.4$ & $4.8 \pm 1.5$ & $4.9 \pm 1.5$ & $5.1 \pm 1.7$ & $4.8 \pm 1.4$ & $4.6 \pm 1.7$ & $4.5 \pm 1.8$ & $4.4 \pm 1.8$ \\
\hline $\operatorname{PAP}\left(\mathrm{cmH}_{2} \mathrm{O}\right)$ & $18.0 \pm 3.0$ & $18.2 \pm 3.0$ & $18.5 \pm 3.0$ & $19.1 \pm 3.4$ & $18.0 \pm 3.0$ & $17.9 \pm 3.0$ & $17.8 \pm 3.0$ & $17.6 \pm 2.6$ \\
\hline $\mathrm{MBP}(\mathrm{mmHg})$ & $83.0 \pm 15.2$ & $77.9 \pm 11.9$ & $78.1 \pm 13.0$ & $77.8 \pm 12.3$ & $83.0 \pm 15.2$ & $76.7 \pm 12.3$ & $76.5 \pm 14.3$ & $77.0 \pm 17.0$ \\
\hline $\mathrm{HR}(/ \mathrm{min})$ & $67.2 \pm 13.4$ & $64.0 \pm 11.8$ & $64.7 \pm 10.9$ & $64.1 \pm 10.5$ & $67.2 \pm 13.4$ & $65.2 \pm 12.1$ & $65.2 \pm 11.9$ & $65.9 \pm 12.8$ \\
\hline
\end{tabular}

Data are presented as means \pm SD. SVV was decreased at slope of operating table of $-5^{\circ},-10^{\circ}$ and $-15^{\circ}$, it was increased at slope of operating table of $5^{\circ}, 10^{\circ}$ and $15^{\circ}(\mathrm{P}=0.000)$. CO and PAP were increased at slope of operating table of $-5^{\circ},-10^{\circ}$ and $-15^{\circ}$, they were decreased at slope of operating table of $5^{\circ}, 10^{\circ}$ and $15^{\circ}(\mathrm{P}=0.045,0.027)$. SVV: stroke volume variation, CO: cardiac output, PAP: peak airway pressure, MBP: mean blood pressure and HR: heart rate. $\mathrm{T}_{0}$ : at $0^{\circ}$ slope of an operating table in supine position, $\mathrm{T}_{-5}$ : at $-5^{\circ}$ slope of an operating table in Trendelenburg positions. $\mathrm{T}_{-10}$ : at $-10^{\circ}$ slope of an operating table in head-down tilt, and $\mathrm{T}_{-15}$ : at $-15^{\circ}$ slope of an operating table in Trendelenburg positions. $\mathrm{T}_{5}$ : at $5^{\circ}$ slope of an operating table of $-5^{\circ}$ in Trendelenburg positions, $\mathrm{T}_{10}$ : at $10^{\circ}$ slope of an operating table in Trendelenburg positions, and $\mathrm{T}_{15}$ : at $15^{\circ}$ slope of an operating table in head-up tilt. 
Perioperative adequate fluid management optimized CO, enhanced perfusion and oxygen delivery to vital organs and traumatized tissue, and it also promoted the wound healing. This eventually led to the decreased rate of admission to intensive care unit, shorter hospital stay, and decreased postoperative morbidity $[1,9,10]$. Thus, this is essential for evaluating the patient's volume status in the field of anesthesiology.

However, it is difficult to evaluate the intravascular volume. Most clinicians still depend on clinical parameters such as blood pressure, heart rate, urine output, and estimated blood loss. In addition, traditional methods for evaluating the intravascular volume such as CVP and PCWP showed lower sensitivity and specificity, and they are not useful in predicting the fluid responsiveness [2,11]. Furthermore, invasive monitoring with pulmonary artery catheterization has been replaced by less invasive methods due to poor outcomes $[12,13]$. The volumetric parameters such as corrected flow time of esophageal doppler and ejection fraction or end-diastolic volume of echocardiography were found to be useful $[14,15]$; but they are disadvantageous because continuous monitoring is unavailable.

The SVV is one of the functional dynamic parameters that may be useful for the fluid management, which is based on heart-lung interaction. That is, in positive pressure ventilation, ITP is increased during inspiration that squeezes blood from the pulmonary capillary and vein into the left heart and augments left ventricle (LV) preload which in turn increases SV. But the pulmonary venous system is soon emptied and the right ventricle (RV) preload is decreased, due to the decreased venous return and the increased RV afterload. This results in delayed reduction of SV. These effects were intensified in volume depletion, so the variability of SV is correlated with fluid responsiveness. That is, the high variation in SV reflects hypovolemia, and SVV greater than $13 \%$ provides responsiveness to fluid therapy [16].

Unfortunately, SVV is subjected to the respiratory factors. It shows poor reliability in patients with poor lung compliance, excessively low tidal volume, or excessively high respiratory rate $[16,17]$.

To date, several studies have been conducted in clinical settings that altered respiratory factors. According to animal studies, the SVV was higher in the increased intra-abdominal pressure, because the venous return was decreased from the caval vein compression [18]. Moreover, the SVV has a poor predictive value for the fluid responsiveness $[19,20]$. In patient placed in prone position, the SVV is increased due to the increased intraabdominal pressure and the decreased respiratory compliance [21]. In patient undergoing laparoscopic surgery, it also has a poor predictive value for the fluid responsiveness because the ITP is affected by the iatrogenic pneumoperitoneum [22,23]. According to another study, however, the SVV has a good pre- dictive value for the fluid responsiveness in patients undergoing laparoscopic surgery in the T position [6]. These recent reports have conflicting results of the SVV and we have encountered more frequent intraoperative changes in the patient posture which might affect the ITP. This is notable in patients placed in the $\mathrm{T}$ or RT position.

When placed in the $\mathrm{T}$ position, patients are characterized by the stiffness of the abdominal part of the chest wall and the cephalad shift of the diaphragm. This is aggravated due to additional surgical retractions. Therefore, it is expected that there might be a decrease in the RV ejection due to the increased ITP and RV afterload. On other hand, such patients are characterized by the increased venous return, thus possibly presenting with the increased RV output.

Our results showed that the SVV was decreased in the patients placed in the $\mathrm{T}$ position. We maintained the volume status consistently throughout the study, but we found that there were changes in the SVV. Presumably, this might be due to differences in the venous return from posture changes, although there was secondary increase in the PAP. It is probable that the PAP might have an effect on SVV to a smaller extent as compared to the increased venous return. This is because the airway driving pressure of $<20 \mathrm{cmH}_{2} \mathrm{O}$ was not significant to transmit to the intracardiac cavity [24]. In the current study, the mean PAP was under $20 \mathrm{cmH}_{2} \mathrm{O}$.

Our results are consistent with other studies showing increases in the RV filling pressure, the RV ejection fraction, and the LV preload, which lead to increased CO on transesophageal echocardiography of patients placed in the T position [7].

It has also been reported that the SVV was increased in patients placed in the RT position. Presumably, this might be due to venous pooling towards the lower extremities [25]. In such patients, there are decreases in the LV end diastolic area and cardiac index on transesophageal echocardiography [26].

There are several limitations of the current study as discussed below.

First, we failed to enroll a homogenous population of the patients. Their median age was 65 years and $66 \%$ of them were men. In addition, 31 patients belonged to the ASA class II, and $52 \%$ of them were taking anti-hypertensive medications. Also, $23 \%$ of our cases had diabetes mellitus patients, although we enrolled the patients whose blood pressure and glucose level were strictly controlled within the normal limit for at least 3 months. We failed to rule out some of the factors regarding the age, sex, and undiagnosed vascular complications. However, in general, most clinicians monitor SVV in patients with critical illness or major operations, so we had no choice but to conduct this study with them. Also, SVV has been reported with good predictive value for the fluid responsiveness even in patients with coronary occlusive disease [27]. Even in patients undergoing carotid 
endarterectomy who had peripheral vascular disease, SVV was a useful predictor for the fluid responsiveness [4]. Therefore, we assume that there was no significant effect in the enrolled patients with ASA classes II.

The results of MBP and HR could be understood in the same context. Because the enrolled patients were aged and about half of them were taking anti-hypertensive drugs, physiologic changes in the blood pressure and HR might have been blunted after postural changes.

Secondly, it remains questionable whether the vasodilatory effects of anesthetic agents in lowering the preload could be maintained consistently throughout the study. To resolve this, we conducted the current study based on the same protocol of anesthesia, and then maintained the BIS score at equivalent with the adjustment of sevoflurane. We also excluded the patients whose hemodynamic data were not maintained within $20 \%$ range, after undergoing measurement three times in a supine position $\left(\mathrm{T}_{0}, \mathrm{~T}_{0}, \mathrm{~T}_{0}\right.$ ”).

Thirdly, there are many surgical positions such as decubitus, prone, sitting position, and so on. Our study was only focused on the patients with T or RT position, so further studies are needed to evaluate the effects on other positions and with pneumoperitoneum, as well.

Lastly, the sample size was small, and we did not evaluate the changes in SVV depending on the patients' postures after dividing the patients into two groups of the responder and nonresponders, after fluid loading. Therefore, we did not assay predictive value of SVV.

In conclusion, our results indicate that the SVV is subjected to the posture in the same patient with the same volume status. This leads to the speculation that clinicians should interpret it after confirming the patient posture before initiating the fluid management or inotropic therapy. Further studies are warranted to evaluate the reliability and predictive values of SVV for predicting the fluid responsiveness with postural change, in larger sample size of healthy patients or specific patient groups with heart or pulmonary diseases.

\section{References}

1. Donati A, Loggi S, Preiser JC, Orsetti G, Münch C, Gabbanelli V, et al. Goal-directed intraoperative therapy reduces morbidity and length of hospital stay in high-risk surgical patients. Chest 2007; 132: 1817-24.

2. Michard F, Teboul JL. Predicting fluid responsiveness in ICU patients: a critical analysis of the evidence. Chest 2002; 121: 2000-8.

3. Zhang Z, Lu B, Sheng X, Jin N. Accuracy of stroke volume variation in predicting fluid responsiveness: a systematic review and metaanalysis. J Anesth 2011; 25: 904-16.

4. Kim KM, Gwak MS, Choi SJ, Kim MH, Park MH, Heo BY. Pulse pressure variation and stroke volume variation to predict fluid responsiveness in patients undergoing carotid endarterectomy. Korean J Anesthesiol 2013; 65: 237-43.

5. Kim HK, Pinsky MR. Effect of tidal volume, sampling duration, and cardiac contractility on pulse pressure and stroke volume variation during positive-pressure ventilation. Crit Care Med 2008; 36: 2858-62.

6. Chin JH, Lee EH, Hwang GS, Choi WJ. Prediction of fluid responsiveness using dynamic preload indices in patients undergoing robotassisted surgery with pneumoperitoneum in the Trendelenburg position. Anaesth Intensive Care 2013; 41: 515-22.

7. Haas S, Haese A, Goetz AE, Kubitz JC. Haemodynamics and cardiac function during robotic-assisted laparoscopic prostatectomy in steep Trendelenburg position. Int J Med Robot 2011; 7: 408-13.

8. Perilli V, Sollazzi L, Modesti C, Sacco T, Bocci MG, Ciocchetti PP, et al. Determinants of improvement in oxygenation consequent to reverse Trendelenburg position in anesthetized morbidly obese patients. Obes Surg 2004; 14: 866-7.

9. Chappell D, Jacob M, Hofmann-Kiefer K, Conzen P, Rehm M. A rational approach to perioperative fluid management. Anesthesiology 2008; 109: 723-40.

10. Cannesson M. Arterial pressure variation and goal-directed fluid therapy. J Cardiothorac Vasc Anesth 2010; 24: 487-97.

11. Kumar A, Anel R, Bunnell E, Habet K, Zanotti S, Marshall S, et al. Pulmonary artery occlusion pressure and central venous pressure fail to predict ventricular filling volume, cardiac performance, or the response to volume infusion in normal subjects. Crit Care Med 2004; 32: 691-9.

12. Sandham JD, Hull RD, Brant RF, Knox L, Pineo GF, Doig CJ, et al. A randomized, controlled trial of the use of pulmonary-artery catheters in high-risk surgical patients. N Engl J Med 2003; 348: 5-14.

13. Polanczyk CA, Rohde LE, Goldman L, Cook EF, Thomas EJ, Marcantonio ER, et al. Right heart catheterization and cardiac complications in patients undergoing noncardiac surgery: an observational study. JAMA 2001; 286: 309-14.

14. Lee JH, Kim JT, Yoon SZ, Lim YJ, Jeon Y, Bahk JH, et al. Evaluation of corrected flow time in oesophageal Doppler as a predictor of fluid responsiveness. Br J Anaesth 2007; 99: 343-8.

15. Tousignant CP, Walsh F, Mazer CD. The use of transesophageal echocardiography for preload assessment in critically ill patients. Anesth Analg 2000; 90: 351-5. 
16. McGee WT. A simple physiologic algorithm for managing hemodynamic using stroke volume and stroke volume variation: physiologic optimization program. J Intensive Care Med 2009; 24: 352-60.

17. Hofer CK, Cannesson M. Monitoring fluid responsiveness. Acta Anaesthesiol Taiwan 2011; 49: 59-65.

18. Jacques D, Bendjelid K, Duperret S, Colling J, Piriou V, Viale JP. Pulse pressure variation and stroke volume variation during increased intra-abdominal pressure: an experimental study. Crit Care 2011; 15: R33.

19. Renner J, Gruenewald M, Quaden R, Hanss R, Meybohm P, Steinfath M, et al. Influence of increased intra-abdominal pressure on fluid responsiveness predicted by pulse pressure variation and stroke volume variation in a porcine model. Crit Care Med 2009; 37: 650-8.

20. Duperret S, Lhuillier F, Piriou V, Vivier E, Metton O, Branche P, et al. Increased intra-abdominal pressure affects respiratory variations in arterial pressure in normovolaemic and hypovolaemic mechanically ventilated healthy pigs. Intensive Care Med 2007; $33: 163-71$.

21. Biais M, Bernard O, Ha JC, Degryse C, Sztark F. Abilities of pulse pressure variations and stroke volume variations to predict fluid responsiveness in prone position during scoliosis surgery. Br J Anaesth 2010; 104: 407-13.

22. Høiseth LØ, Hoff IE, Myre K, Landsverk SA, Kirkebøen KA. Dynamic variables of fluid responsiveness during pneumoperitoneum and laparoscopic surgery. Acta Anaesthesiol Scand 2012; 56: 777-86

23. Bliacheriene F, Machado SB, Fonseca EB, Otsuke D, Auler JO Jr, Michard F. Pulse pressure variation as a tool to detect hypovolaemia during pneumoperitoneum. Acta Anaesthesiol Scand 2007; 51: 1268-72.

24. Muller L, Louart G, Bousquet PJ, Candela D, Zoric L, de La Coussaye JE, et al. The influence of the airway driving pressure on pulsed pressure variation as a predictor of fluid responsiveness. Intensive Care Med 2010; 36: 496-503.

25. Buhre W, Weyland A, Buhre K, Kazmaier S, Mursch K, Schmidt M, et al. Effects of the sitting position on the distribution of blood volume in patients undergoing neurosurgical procedures. Br J Anaesth 2000; 84: 354-7.

26. Rex S, Brose S, Metzelder S, Hüneke R, Schälte G, Autschbach R, et al. Prediction of fluid responsiveness in patients during cardiac surgery. Br J Anaesth 2004; 93: 782-8.

27. Wuffels PA, Sergeant P, Wouters PF. The value of pulse pressure and stroke volume variation as predictors of fluid responsiveness during open chest surgery. Anaesthesia 2010; 65: 704-9. 